\title{
Nueva especie de Hydraena Kugelann, 1794 del Parque Nacional de la Sierra de Cazorla, Jaén, España (Coleoptera : Hydraenidae)
}
A. Lagarl
X. Fresneda ${ }^{2}$
C. Hernando ${ }^{3}$

\begin{abstract}
Nouvelle espéce d̀ Hydraena Kugelanm, 1794 du Parc National de la Sterra de Cazorla, Jaén, Espagne. (Coleoptera : Hydraenildae)
Mots clés : Coleoptera, Hydraenidae, nova species, Sierra de Cazorla, Espagne.

Ce travail décrit Hydraena quilisi sp. nov. d'Andalousie orientale. Elle se caractérise par un procès dentiforme des métatibias du mále, caractère jamais observé chez aucune autre Hydraena ibérique. Elle présente d'étroites relations avec des espèces d'Italie mais la structure spéciale du parmètre droit de son édéage, très dilaté à l'extrémité, est bien différente de celles de ces espèces voisines (groupe $H$. fiorii Porta, 1099 et $H$. imperainix Kniz, 1919).
\end{abstract}

\begin{abstract}
A new species of Hydraena Kugelann, 1794 from the National Park of Sierra de Cazorla, Jaén, Spatn. (Coleoptera : Hydraenidae)
Keywords : Coleoptera, Hydraenidae, new species, Sierra de Cazorla, Spain.

This publication describes Hydraena quilisi sp. nov. from Eastern Andalusia. The chief character for this species is the tooth-like process on the male metatibia, a character that has never been found in any other Iberian Hydraena species. This species shows some similarities with Italian species but the special structure of the right paramere with the very dilated tip of the aedeagus is very different from those of neighbouring species (group $H$. fiorii Porta 1899 , and $H$. imperatrix Kniz 1919).
\end{abstract}

En este trabajo describimos una interesante nueva especie del género Hydraena de morfología sin precedentes entre las de la Península Ibérica. Agradecemos sinceramente al señor G. Ferro, de Lancenigo (Treviso - Italia), la cesión de un ejemplar o de Hydraena imperatrix Kinz, 1919, que nos ha facilitado en gran parte el estudio de nuestra nueva especie.

\section{Hydraena quilisi nov. sp.}

Holotipo o* : Arroyo de San Pedro de la Garganta, cerca de Nava de San Pedro, dentro del Parque Nacional de la Sierra de Cazorla, Jaén ; figs. 1-6. 8-VIII-1985, C. Hernando y X. Fresneda leg. (en Col. C. Hernando, Barcelona).

\footnotetext{
1. Sanjuanistas, 20, E - 08006 Barcelona, Espana.

2. Manso Pujol, 3, E - 08032 Barcelona, Espana.

3. Cadi, 47, E - 08031 Barcelona, Espana.
}

Alotipo ơ : Capturado junto con el $\sigma^{*}$. (Col. X. Fresneda, Barcelona).

Biometria :

\begin{tabular}{lrc}
\multicolumn{2}{c}{ Longitud (mm) } & Anchura (mm) \\
Holotipo or & 2.35 & 0.92 \\
Alotipo or & 2.16 & 0.86
\end{tabular}

Descripción :

Color marrón oscuro.

Cabeza de proporciones normales; la puntuación del epístoma muy fina ; palpos simétricos, testáceo claro uniforme. Frente con la puntuación formada de puntos gruesos, espaciados, separados por especios unas des veces más anchos que los puntos, de superficie lisa ; en la parte supraocular los puntos son apretados y de aspecto opaco. Ojos salientes, un poco más anchos que el borde anterior del protórax.

Protórax con la máxima anchura en el medio desde donde se estrecha progressivamente hacia los ángulos anteriores, de lados convergentes: hacia el medio subanguloso, redondeado, estrechado hacia 


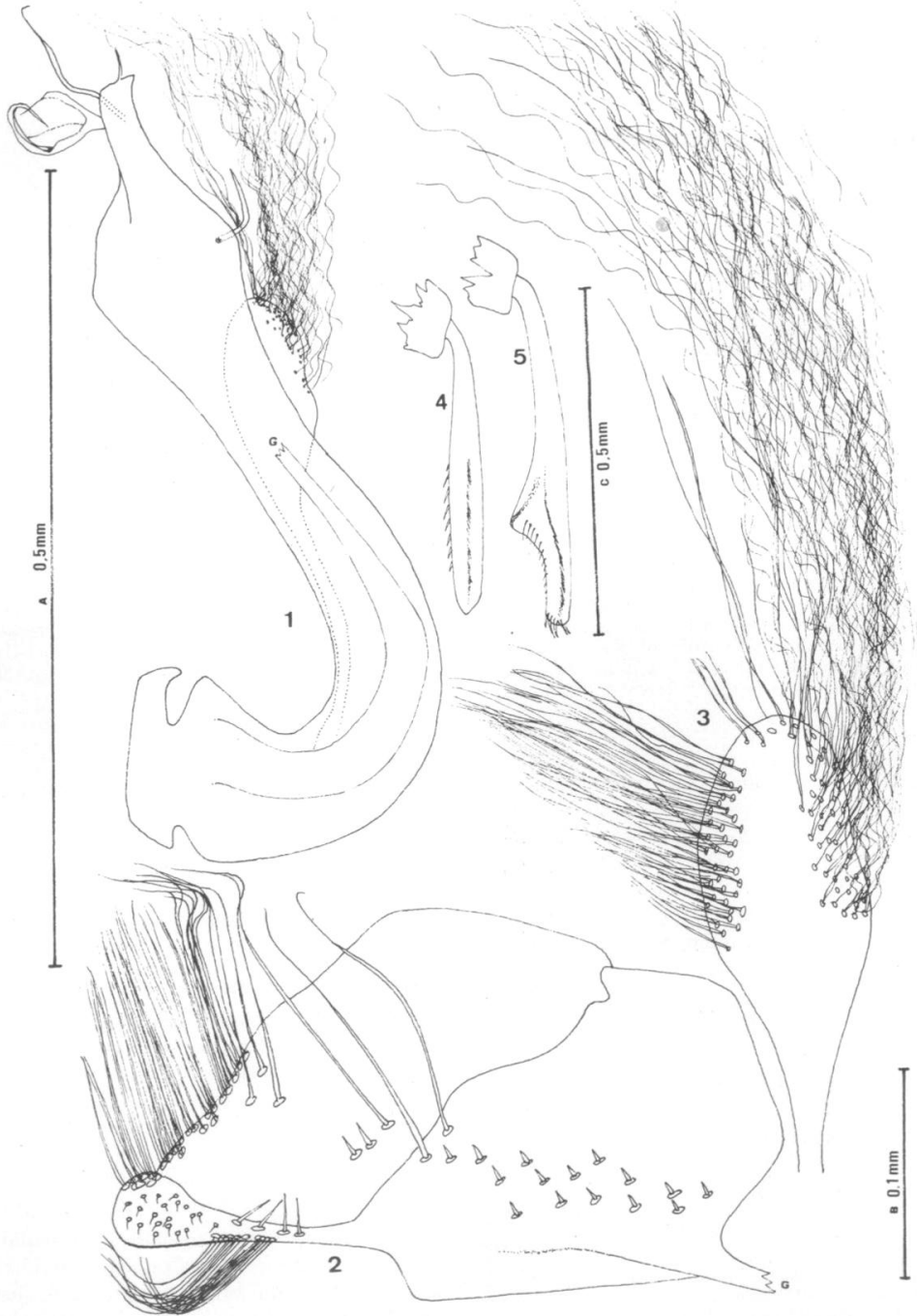

Fig. 1-5: vista lateral del edeago de Hydraena quilisi nov. sp. ‘El ápice del estilo derecho no se ha dibujado porque tapa totalmente las estructuras apicales del pene, en el lugar marcado con una G, pués, va situada la fig. 1); 2 ; ampliación del ápice del estilo derecho 3 : ampliación con detalle de las sedas del ápice del estilo izquierdo $4:$ mesotibia derecha del Holotipo $0 ; 5$, metatibia derecha del Holotipo o . (la escala A corresponde a la fig. 1, la B a las fig. 2 y 3 , y la $\mathrm{C}$ a las fig. 4 y 5 ). 


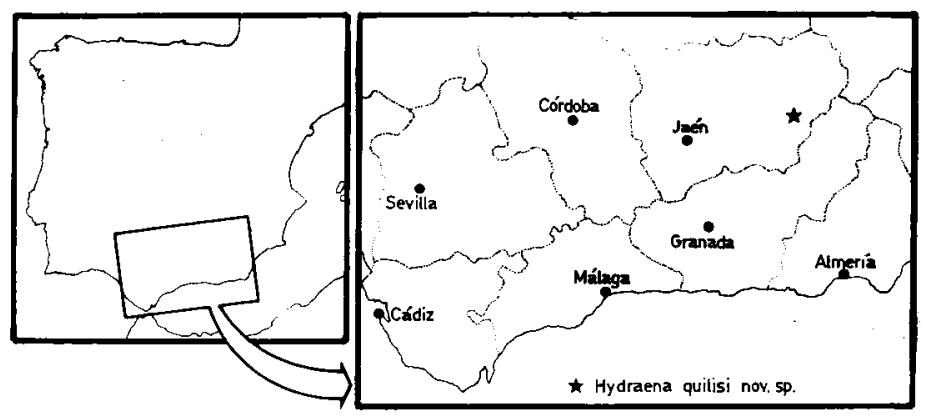

Fig. 6. : Localización de Hydraena quilisi nov. sp.

atrás, con el borde posterior poco más ancho que el anterior. La puntuación es hacia los lados apretada y densa, la del medio gruesa, bien espaciada, de intervalos lisos y más grandes que lospuntos mismos ; la del borde anterior apretada, opaca ; en el borde posterior los puntos son prácticamente de doble diámetro que los del disco, con los intervalos lisos menores que los puntos. Reborde lateral fino. suavemente aserrado al margen.

Elitros con 8-10 series de puntos entre la sutura y el callo humeral, con series regulares, embrollados hacia el ápice, los intervalos más estrechos que los puntos; forma oblonga con la máxima anchura hacia el tercio posterior; los lados caen sobre el margen lateral suavemente, este margen es poco ancho, en forma de canal algo cóncavo. Apice truncado, el ángulo sutural no dentado.

Patas de proporciones normales, más bien robustas ; metatibias or en su tercio distal con un conspicuo proceso dentiforme en el lado interno (fig. 5). Mesotibias $\sigma$ con una serie de espinitas en su lado interno.

Edeago (fig. 1) recuerda algo al de imperatrix Kniz, 1919, en Pirisinu (1981, fig. 11 f), pero el tubo distal es más largo y no ensanchado en el ápice ; pero sobre todo la estructura del parámero derecho y la quetolaxia del mismo no permiten relacionarlo con imperatrix ni ninguna otra especie, el parámero izquierdo corresponde al tipo normal.
Dimorfismo sexual :

$\sigma:$ Metatibias con proceso dentiforme en el tercio distal del borde interno.

\&: Metatibias simples; palpos menos robustos; talla más pequena ; en general de habitus más grácil.

Afinidades :

Esta interesante especie no admite comparación con ninguna $H y d r a e n a$ iberica conocida. Por el notable proceso dentiforme de la metatibia del or se acopla en el conjunto de especies designado por Kuwert en 1888 como subgénero Hoplydraena, ya sinonimi. zado en el catálogo Reitter (1906).

Por su morfología externa se relaciona estrechamente con H. imperatrix Kniz, 1919, de la que copia la forma, color e idéntico proceso dentiforme en la metatibia del $\alpha$. La talla es algo menor, $2-2.20 \mathrm{~mm}$ frente a $2,35 \mathrm{~mm}$ de quilisi, el protórax lateralmente con el aserrado mucho más fino en imperatrix de forma que casi no se percibe a $\times 37.5$ en tanto que en quilisi es bien manifies to a est a magnitud, siendo preciso un minimo de $\times 50$ para apreciarlo en imperatrix.

Asimismo por el proceso dent iforme de la metatibia or se relaciona con $H$. fiorii Porta, 1899 y $H$. spinipes Baudi, 1882 que tiene este proceso diferente en forma ganchuda hacia atrás (Porta 1900, pl. I, fig. 2 y 4), de Italia, pero muy alejada por el edeago absolutamente de otro tipo. 
Derivatio nominis :

En agradecimiento a Mariqueti Garcia Quilis, de Tocina (Sevilla), que durante la campana entomoló gica de agosto de 1985 tuvo la amabilidad de acompanarnoos a numerosos arroyos y charcas que ella conocia en Andalucía.

\section{Addendum}

En prensa nuest ra descripción de Hydraena quilisi recibimos una valiosa comunicación del Dr. C. Berthélemy, que ante todo agradecemos sinceramente, y transcribimos seguidamente :

"Esta especie ha sido recolectada en la alta cuenca del rio Madera (Jaén. Sierra de Segura) y en el río Hoyas (Albacete, cuenca del rio Segura), por el Dr. C. Montes en 1982 y 1983. Asimismo ha sido también recolectada por una alumna del Prof. Alba-Tercedor en la zona alta del río Aguas Blancas (Granada, Sierra Nevada), en octubre y noviembre. Esta captura aparecc citada en una Memoria de Licenciatura de la Universidad de Granada, Facultad de Ciencias, Departamento de Zoología ; se trata de un tra. bajo en multicopia, no impreso, de C.E. Sainz-Cantero
Caparrós : Coleópteros de los cursos de agua de Sierra Nevada: Taxonomia y Ecologia de las familias Elmidae. Dryopidae e Hydraenidat, $237 \mathrm{pp}$. Más interesante y curioso es todaria el que esta misma especie ha sido hallada en Francia, en el curso superior de la Dróme, LucenDróme, el 16.08.1985 (F. Hebauer leg.). En consecuencia, Hydraena quilisi no es endémica de la Peninsula Ibérica ; esta estación constituye por tanto un pasaje con las especies italianas más próximas n(C. Berthélemy 1987, in litt.).

\section{Autores cilados}

Baudi (F.). 1882. Descripción de Hydraena spinipes. Naturista sicil., $1(1881.1882): 130$

Chiesa (A.). 1959. Hydrophilidae Europae (Coleptera Palpicomia): 200 p. A. Forti, Bologna

Kuiz (A.). 1919. Zur Kerntris der palacark tischen Hydrophiliden. Ent. Bl. Biol. Syst. Käfer, $15: 11-16$

Kuwert (A.). 1888. Generalübersicht der Hydraenen der europäischen Fauna. Dt. ent. Z., 32 (1): 113-123.

Porta (A.). 1899. Hydraena (Hoplydraena) fiorit. Miscnea ent., 7 : 29-31

Porta (A.). 1900. Révision du sous-genre Hoplydraena Kuw. Misenea ent., $8(1): 1-3+1$ lám de 6 figs.

Pirisinu (Q.). 1981. Palpicorni (Coleptera : Hydraenidae, Helophoridae, Spercheidae, Hydrochidae, Hydrophilidae, Sphaeridiidae). Guide per il niconoscimento delle specie animali delle acate inteme italiane, 13: 1-97. 